\title{
A Qualitative Analysis of the Diameter of Long Positive Jet Streamer above Thundercloud in Uniform Atmosphere
}

\author{
Avnish Kumar*, Parmanand Prakash Pathak \\ Department of Physics, Gurukul Kangri Vishwavidyalaya, Haridwar, Uttarakhand, India \\ Email: *kumar87avnish@gmail.com
}

How to cite this paper: Kumar, A. and Pathak, P.P. (2021) A Qualitative Analysis of the Diameter of Long Positive Jet Streamer above Thundercloud in Uniform Atmosphere. Journal of Electromagnetic Analysis and Applications, 13, 123-134. https://doi.org/10.4236/jemaa.2021.139009

Received: July 16, 2021

Accepted: September 19, 2021

Published: September 22, 2021

Copyright $\odot 2021$ by author(s) and Scientific Research Publishing Inc. This work is licensed under the Creative Commons Attribution International License (CC BY 4.0).

http://creativecommons.org/licenses/by/4.0/ (c) (i) Open Access

\begin{abstract}
A qualitative analysis of the diameter of the spherical head of a long positive Jet streamer above thundercloud is presented in this paper under uniform atmospheric condition for streamers of less than $7 \mathrm{~km}$ length. In this study, an attempt is made to replicate laboratory-based point electrode discharge model for jet streamers originating above the thunderclouds. In laboratory conditions, it is not possible to produce huge electrode potentials which could be the reason that the streamers generated under the controlled lab environment have diameter of the order of only a few centimeter and length of a few millimeter. On the other hand, the thunderclouds carry huge electrical charges, for example $50 \mathrm{C}$, which can produce huge electrical potentials of the order of several hundred $\mathrm{MeV}$. Such huge potential can act as the potential of a point electrode which may be capable of producing very large and thicker streamers above the thunderclouds. So, a leader mechanism of streamer initiation is assumed in calculations as the tip of conducting leader channel can act as point electrode carrying huge cloud potential to generate large streamers. It is found in this study that as the streamer moves larger distance away from the electrode (leader tip), the diameter of the streamer head decreases. Higher the potential of the electrode (leader tip), thicker is the streamer and more slowly the diameter decreases. Also, it is also found in our calculations that for higher electrode (leader tip) potential lower is the altitude of initiation of streamers.
\end{abstract}

\section{Keywords}

Streamer, Jet, Thunderclouds, Electric Field, Leader

\section{Introduction}

A streamer is a narrow channel of cold plasma giving it low conductivity. The 
electric field near the tip of it increases up to 4 - 7 times the critical field $E_{k}$ [1] which in turn triggers the streamer initiation. Once the streamer is triggered, it advances itself even in a comparatively weak external field. The streamer propagates upward as long as the field at the tip is greater than the critical field required for the propagation of the streamer. At lower atmospheric altitudes, the air density is high giving rise to the process of triple attachment of electron to the atmospheric oxygen prominently which leads to the loss of the conductivity of the streamer rapidly. That is the reason a long spark discharges and lightning is better explained by leader mechanism.

The gas temperature in the streamer channel goes up to $5000 \mathrm{~K}$ or more which maintains the conductivity of the leader channel. In this way, the tip of the leader continuously produces bulk of streamers in the form of bunch of filaments. The fan of streamers form a streamer zone on the front side of leader and the total current warms enough up the leader channel to maintain conductivity. The space charge at the front of the streamers covers the leader channel which in turn reduces the electric field in the lateral surface of the leader stopping the process of ionization, expansion and cooling of the atmospheric air around the leader channel [2] [3].

The negative streamer-leader system requires more than two times the electric field and electric charge than required by positive streamer and leader system. Apart from this, for the initiation of a negative Jet streamer, there should be strong + CG discharge because in this process the direction of electric field above the thundercloud is changed when the positive charge on the top of the cloud is lowered/removed. But it is clear from various observations that the Jets are not linked with a particular polarity of lightning discharges [4]. Moreover, the negative polarity jets impractically require large thundercloud charges. So, this may be the compelling reason behind the assumption of positive Jets polarity. In this way, there exist similarity between the Jets and the streamer zone of leader which was proposed and assumed by several authors [5] [6]. It has been shown that a point base is the source of the filamentary structure and branching pattern in the streamer corona [7].

The electric field generated by the thundercloud charge is self-consistent in which propagation, branching and growth of filamentary structure of streamer zone take place. The ratio of electric field inside the channel of streamer and molecular number density $E_{S} / N$ was assumed to be constant in this approach. Here the molecular number density is described by $N \propto \exp (-h / \Delta), \Delta$ (scale height of atmosphere) is of the order of $7 \mathrm{~km}$.

But there are some shortcomings in the above discussed approach. The first flaw is disregarding sharp decrease of conductivity of the channel of streamer in the fractal model studies. In fact, under normal atmospheric conditions streamer zone of a leader extends up to a few meters while the length of individual channels of higher conductivity constituted behind the streamer tip is of the order of centimeter level [2]. This is the main reason restricting the electric current 
supply from lower branches of fractal trees to the top branches. Another shortcoming of the fractal model studies is that these lack an effective charge transport mechanism which is attached with the ice particles, water droplets and hails pervading throughout the volume of the thundercloud. There are similar other drawbacks which forced some scholars to assume bi-leader model of conventional lightning [3]. However, the thundercloud charge remains intact and unaffected and it generates the required electric field.

Raizer et al. [8] suggested a Jet model which was based on leader mechanism of streamers formation. For a cylindrical streamer, the maximum electric field at the tip of the streamer is approximately half of the ratio of its tip potential and the radius of its spherical head [9]. The tip/front of the positive streamer advances upward from the initiation point as long as the electric field in the vicinity of the tip is higher than the threshold electric field. Thus, a potential gradient equal to the product of inside electric field and length of streamer is developed along the streamer. One can assume the uniformity of atmosphere for streamers less than $7 \mathrm{~km}$ length. The electric field at the front of the streamer becomes maximum which fades away towards the edges [10]. This field can be $4-7$ times higher than the threshold field and when it becomes less than the threshold value streamers stops propagating.

In laboratory experiments, streamers are generated in gas discharge tube by a high electric potential point electrode. These streamers have diameter of the order of few millimeter and length of the order of several centimeter [11]. The thickness of streamers and their length depends on the electrode voltage and its thickness. For, thicker electrode and higher electrode potentials the diameter of streamers is thicker while the decline rate of diameter is slower [11]. Since, it is not possible to generate huge electrode potential in laboratory conditions, the streamers generated in such environment are of the order of few centimeter.

But, thunderclouds carry large amount of charges capable of producing huge potentials which can be a source of large streamers originating above thunderclouds. So, with the help of a suitable mechanism, it is quite possible to explain the reason behind the large streamers originating above the thunderclouds. So, in this study, a leader to streamer mechanism is adopted in which it is assumed that first a leader propagates from the top surface of thunderclouds carrying huge potential of cloud surface which can act as the point electrode to generate fan of streamers. This consideration has additional advantage of bypassing the requirement of large impractical thundercloud charges of 120 - $130 \mathrm{C}$ [6] for triggering streamers. In this mechanism, the potential required for streamer initiation is delivered by the leader from the cloud surface. In this paper, the variation of streamer head diameter with its length at thundercloud level is studied and compared with the results under laboratory conditions.

\section{Theoretical Model}

In this paper, theoretical model is explored in which the point electrode corona 
discharge experiment conducted under laboratory conditions is replicated in the atmospheric condition above thunderclouds level as shown in Figure 1. It was considered in previous theories that the upward moving jets (Blue Jets) appearing above thunderclouds are similar to the streamers growing in the point electrode corona discharge experiments conducted in the laboratory environment [12] [13]. Moreover, Pasko and George [6] suggested that an irregularity of positive charge with lateral dimension of about $1 \mathrm{~km}$ at the top surface of the thundercloud can act as a triggering point for the initiation of Jets [12]. This positive irregularity at the upper surface of thundercloud can serve the same purpose as the point electrode does in the corona discharge experiments conducted in laboratory. The upward moving (towards ionosphere) positively charged front of Jet streamers can be treated as the plane electrode (Figure 1). Based on the charge distribution suggested by Krehbiel et al. [14], inside thunderclouds, it is assumed in this theoretical study that there exists a spherical irregularity of positive charge of around $3 \mathrm{~km}$ radius $(R)$ which is located at approximately $12 \mathrm{~km}$ altitude $(Z)$ near the top of the thundercloud above the ground. In the calculations made in this paper two uniform positive charge distribution possibility of $40 \mathrm{C}$ and $50 \mathrm{C}$ are taken into account over a sphere of $3 \mathrm{~km}$ radius. The Jets generally appear at $17-18 \mathrm{~km}$ atmospheric altitudes. But at these altitudes a $40-50$ $\mathrm{C}$ charge distribution is insufficient to generate electric field required to initiate Jet streamers. This may be the reason which compelled Pasko and George [6] to unrealistically consider 120 - $130 \mathrm{C}$ positive charge at the thunderclouds in their fractal model studies.

But in the present study leader to streamer generation mechanism is taken into account in which it is assumed that initially a leader channel originates from the top surface of the positively charged spherical irregularity located in the thundercloud which carries potential of the cloud surface along the leader tip. The head of leader channel advances up to $3 \mathrm{~km}$ in the upward direction from the top surface of cloud located at the $14 \mathrm{~km}$ altitude after which streamer zone is formed as shown in Figure 1. The streamer zone is a system of large number of streamers having low conducting cold plasma channels. So, there should be a voltage gradient along the streamer zone as suggested by Allen N L and Ghaffar A [15]. In this approach, the requirement of unrealistically large thundercloud charge of $120 \mathrm{C}-130 \mathrm{C}$ is eliminated for the initiation of streamers because the conducting leader channel carries huge thundercloud potential to the head of the leader to build the required voltage along the streamer zone of the leader.

\section{The Electric Field at the Streamer Head}

The head/tip of the streamer is sketched in Figure 2(a). As per previous studies, the space charge on the head of a positive streamer remains distributed in the sphere having radius $R_{S}$ [9]. So, it can be concluded that the electric field ahead of the streamer head must decrease quadratically. 


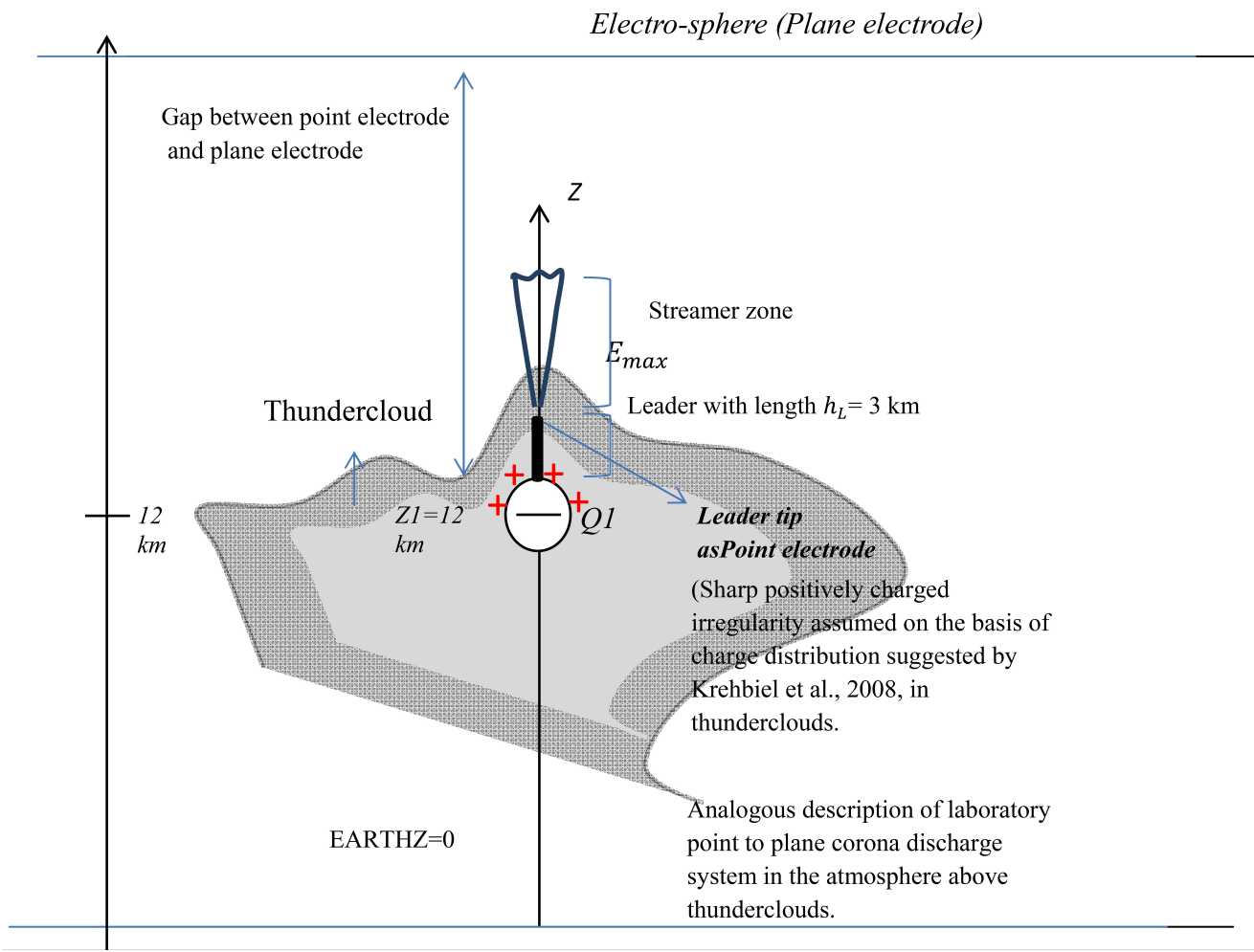

Figure 1. Analogy of laboratory-based point electrode corona discharge model above thunderclouds.

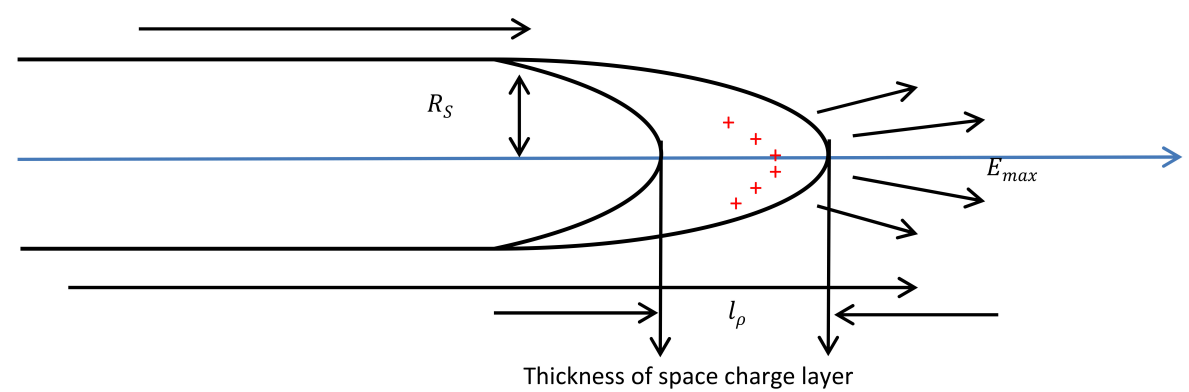

(a)

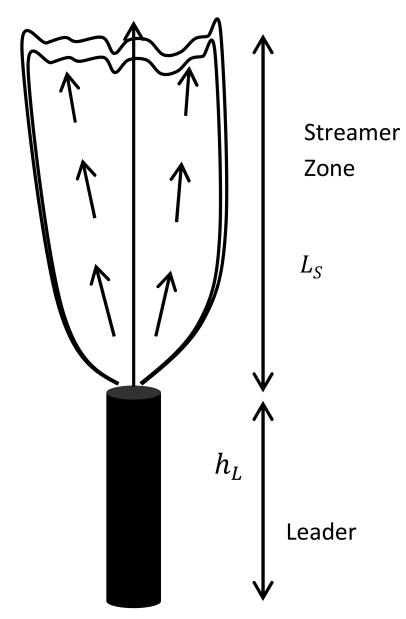

(b)

Figure 2. (a) Structure of positive streamer head [18]; (b) leader and streamer zones of a typical Jet [8]. 
But, the numerical results of Kulikovsky [16] shows that the electric field produced by the space charge of the head of streamer tip does not follows Coulomb's law of inverse square. A better and logical approximation of space charge electric field is presented by Kulikovsky [17] as follows;

$$
E(x)=E_{\max } /\left(1+\frac{x-l_{\rho}}{2 l_{\rho}}\right) \quad x>l_{\rho}
$$

where $l_{\rho}$ is space charge region thickness, typically 2 to 3 times smaller than the radiating radius $R_{S}$ [10] [18]. The ratio of $R_{S} l_{\rho}=3$ is used by Chen et al. [11] in their calculations. So, Equation (1) can be expressed as

$$
E(x)=E_{\max } /\left(1+\frac{1.5\left(x-\frac{R_{S}}{3}\right)}{R_{S}}\right)
$$

here, $E_{\max }$ is the maximum electric field at the spherical head of the streamer which, however, is not the same as the maximum electric field of an isolated sphere. Pancheshnyi et al. [9] estimated that $E_{\max }$ is can be expressed as $U / 2 R_{s}$ where $U$ is the potential of streamer head. As the streamer channel continues to expand, the electric field at the edge of its cylindrical surface goes on decreasing. In this way, $E_{\max }$ can be expressed as given below [10]:

$$
E_{\max }=\frac{U}{R_{S} \ln \left(\frac{L}{R_{S}}\right)}=U / n R_{s}
$$

where, $n$ varies between 2 to 4 .

\section{Relation between Diameter and Length of Streamer in Uniform Atmosphere-}

The peak electric field at the spherical head of the streamer is expressed in Equation (3). Now, in the course of upward propagation, originating from the positively charged spherical irregularity near the top of thundercloud, let the leader moves a distance of $h_{L}$ (Figure 2(b)) and carries cloud potential $U_{c}$ along with the leader tip. In this way, the cloud potential $U_{c}$ becomes equal to the potential of the leader tip $U_{L}$ as shown in Figure 2(b) because the leader channel is considered perfectly conducting to form equipotential surface. If $L_{S}$ be the streamer channel length and $E_{S}$ be the electric field inside the streamer channel, the potential drop along the streamer zone must be given by the following expression-

$$
E_{S} L_{S}=U_{L}-U_{O}\left(h_{L}+L_{S}\right)
$$

where $U_{L}$ is the leader tip potential at $h_{L}$ above top of the thundercloud and $U_{O}\left(h_{L}+L_{S}\right)$ is the potential of the upward moving front of the streamer zone of length $L_{s}$.

If $Q_{C}$ be the positive charge distributed over spherical irregularity of radius $R$ in the thunderclouds producing electric field $E_{S}$ the leader tip potential and the potential of the moving front of streamer zone can be written as 


$$
U_{C}=U_{L}=Q_{C} / 4 \pi \varepsilon_{0} R, U_{O}\left(h_{L}+L_{S}\right)=U_{C} R /\left(R+h_{L}+L_{S}\right)
$$

The relation between the potential of the moving front of the streamer zone and the maximum electric field $E_{\max }$ is given by Equation (3). Thus, at the terminating altitude of the streamer of length $L_{\mathcal{S}}$ the following condition must be satisfied

$$
E_{\text {max }} \approx E_{S} \approx 500 \times \exp \left(-\frac{z}{\Delta}\right) \mathrm{kV} / \mathrm{m}, \text { where } \Delta \approx 7 \mathrm{~km} \quad \text { [2] }
$$

Therefore, under the condition of uniform atmosphere, streamer length less than $7 \mathrm{~km}$ [8] Equations (3)-(6) can be combined to get the following expression

$$
R_{s}=\frac{U_{C}}{n E_{S}}-L_{S} / n
$$

\section{Qualitative Analysis of Results and Discussion-}

In calculations performed in this paper are based on Equation (7). A positively charged spherical irregularity of around $3 \mathrm{~km}$ radius $(R)$ located at $12 \mathrm{~km}$ altitude near the top of the thundercloud is taken into account in performing calculations. Since, the Jet streamers are most often observed at an altitude of around $18 \mathrm{~km}$, the leader channel length $h_{L}$ is taken about $3 \mathrm{~km}$ [8]. For a leader channel of $3 \mathrm{~km}$ length, the head of the channel responsible for triggering streamers would be at $18 \mathrm{~km}\left(12+R+h_{L}\right)$ altitude above the ground. In the calculations made in this paper, $40 \mathrm{C} \& 50 \mathrm{C}$ positive cloud charge $\left(Q_{C}\right)$ distributions are taken into consideration as suggested by Krehbiel et al. [14]. For a given charge configuration, three values of $n(2,2.2 \& 2.4)$ of Equation (3) are taken into consideration. Under assumption of uniform atmosphere for streamers of less than $7 \mathrm{~km}$ length, the graphical variation of positive streamer head diameter is plotted against its length using Equation (7). The results are shown in Figures 3-6.

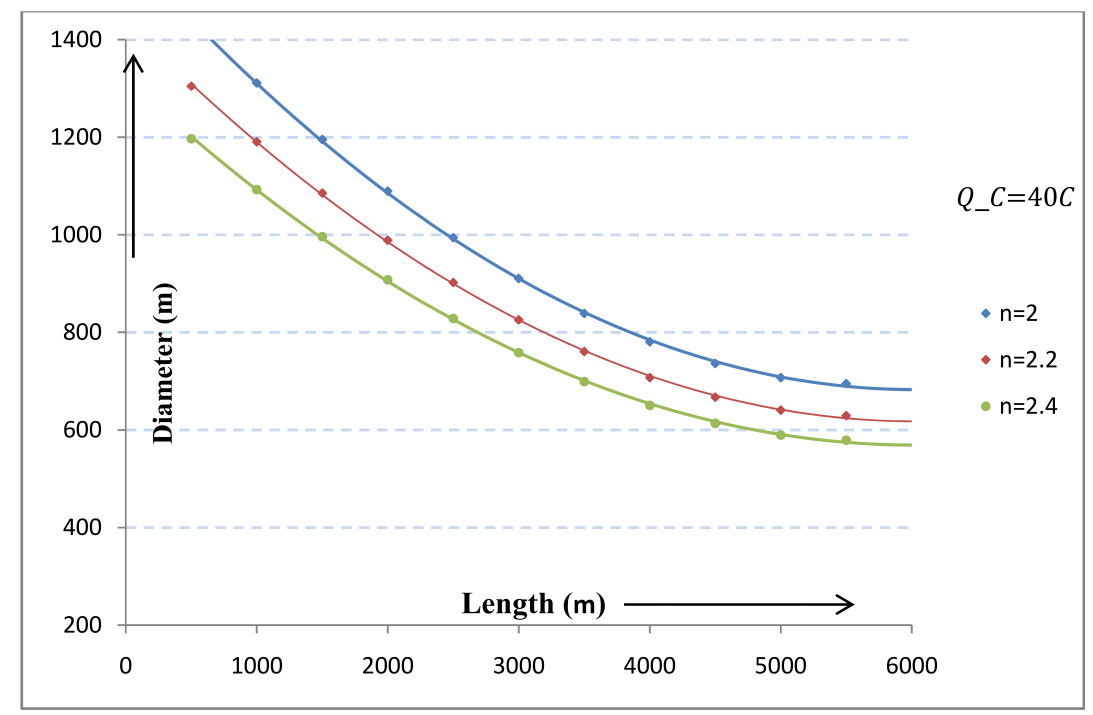

Figure 3. Streamer head diameter (m) vs. the length (m) of positive Jet streamer for a positive cloud charge of $40 \mathrm{C}$. 


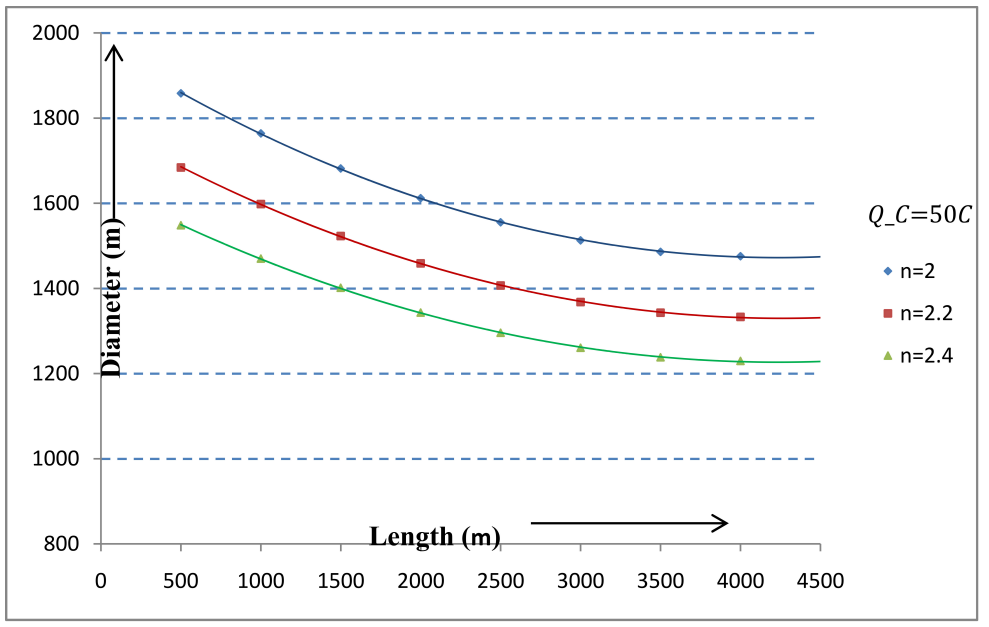

Figure 4. Streamer head diameter (m) vs. the length (m) of positive Jet streamer for a positive cloud charge of $50 \mathrm{C}$.

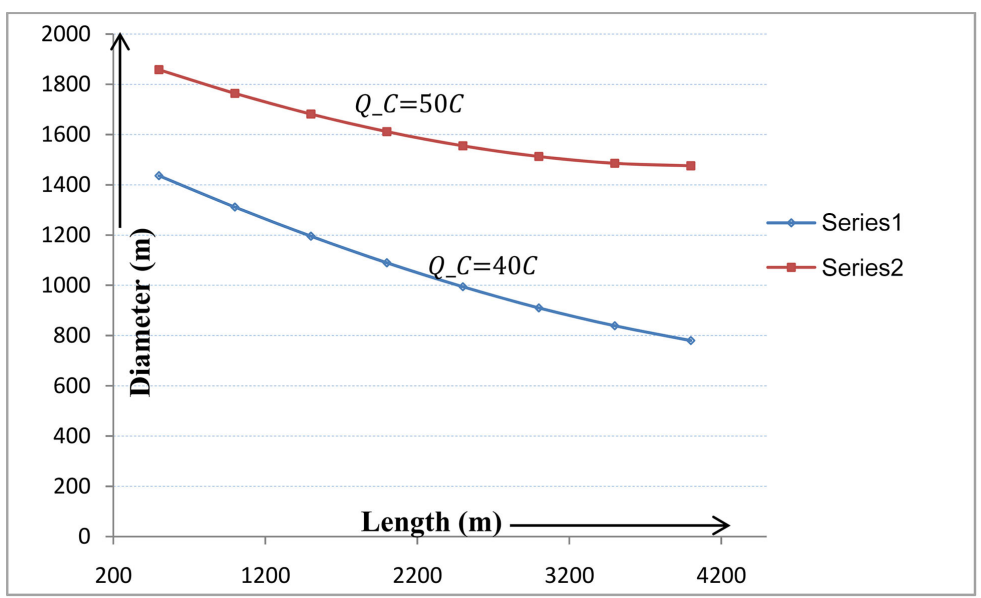

Figure 5. Streamer head diameter $(\mathrm{m})$ vs. the length $(\mathrm{m})$ of positive Jet streamer for a positive cloud charges $40 \mathrm{C}$ of $50 \mathrm{C}$.

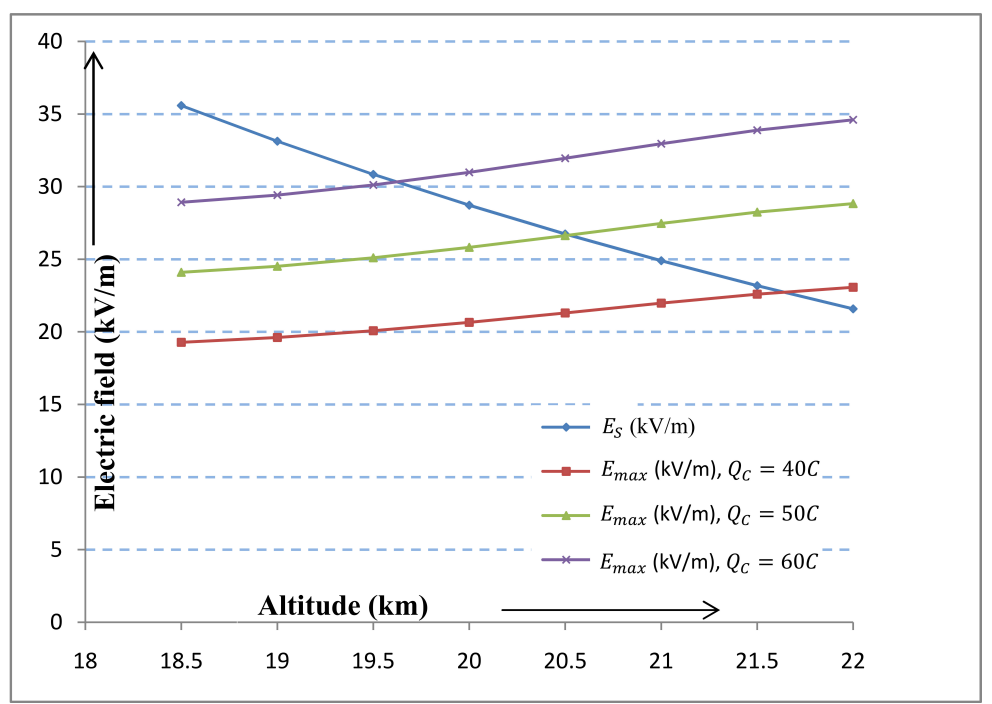

Figure 6. Electric field $(\mathrm{kV} / \mathrm{m})$ at the head of streamer vs. altitude $(\mathrm{km})$ for positive cloud charge of $40 \mathrm{C}, 50 \mathrm{C}$ and $60 \mathrm{C}$. 
Figure 3 \& Figure 4 shows that the streamer head diameter decreases with its length for both cloud charge configurations of $40 \mathrm{C} \& 50 \mathrm{C}$ generating leader tip potential. Moreover, larger the value of parameter $n$ of Equation (3), more quickly the head diameter decreases with the length of streamer. Additionally, from the comparison of for Figure 3 \& Figure 4 it is clear that the bigger the amount of cloud charge distribution, larger will the electrode potential i.e. leader tip potential and thereby greater is the streamer head diameter which comparatively decreases slowly with its length. So, the thickness and variation of streamer head diameter depends on the amount of charge configuration and thereby on the electrode/leader tip potential.

The graph between streamer head diameter and its length is plotted in Figure 5 for positive cloud charges of $40 \mathrm{C}$ and $50 \mathrm{C}$ which clearly demonstrate the results discussed above. The results drawn in this study are in good agreement and backed by the results of previous theoretical studies and experimental studies conducted under laboratory environment under lightning impulse voltage [11].

The electric field $\left(E_{S}\right)$ expressed in Equation (6) and required for triggering streamers above altitude of $18 \mathrm{~km}$ is also plotted (Figure 6) against the peak value of electric field $\left(E_{\max }\right)$ expressed in Equation (7) at the upward moving front of the positive streamers for three cloud charge distribution configurations of $40 \mathrm{C}, 50 \mathrm{C}$ and $60 \mathrm{C}$ generating different electrode/leader tip potentials for streamer initiation. The electric field required for upward propagation of the positive streamers originating above thunderclouds falls exponentially with the atmospheric altitude [2]. But, the maximum electric field at the upward moving spherical streamer head increases with the atmospheric altitude [3] and becomes equal to the electric field expressed in Equation (6), at a particular altitude and for a given leader head potential. Thus, it can be concluded from the comparative study of graph plotted in Figure 6 that the larger the thundercloud charge larger will be the electrode/leader head potential and thereby lower is the streamer initiation altitude. This conclusion is also supported by several previous studies [6] which is obvious as the higher thundercloud charge can comparatively produces strong enough electric field even at lower altitudes to trigger streamers followed by the breakdown of the atmospheric air.

\section{Conclusion}

In this paper, a theoretical investigation of qualitative analysis of the variation of spherical shaped diameter of spherical positive streamer head with the length of the streamer above thunderclouds considering uniform atmosphere assumption. The theoretical model adopted in this study is exactly similar to the point electrode corona discharge model having been studied in laboratory environment. It is assumed in this paper that a positively charged spherical inhomogeneity is located at the top of the thundercloud which may be treated as a point electrode. A leader is initiated from the spherical surface of the cloud irregularity which delivers the strong potential to the tip of the leader to generate positive Jet strea- 
mers at around $18 \mathrm{~km}$ altitudes. The calculations are made accordingly and it is concluded that as the head of positive streamer moves upward originating from the leader tip/cloud surface which acts as point electrode, the diameter of its head decreases gradually. Another conclusion that can be drawn out of this study is that the thickness of streamer head depends on the electrode (cloud) potential. Moreover, for higher leader (acting as point electrode) potential, the streamer head diameter decreases comparatively and triggering altitude of streamers generation is also low for higher electrode/cloud potentials. The findings in this study are in good agreement and comply with the previous observations based on the laboratory experiments under lightning impulse voltage.

\section{Conflicts of Interest}

The authors declare no conflicts of interest regarding the publication of this paper.

\section{References}

[1] Celestin, S. and Pasko, V.P. (2010) Effects of Spatial Non-Uniformity of Streamer Discharges on Spectroscopic Diagnostics of Peak Electric Fields in Transient Luminous Events. Geophysical Research Letters, 37, Article ID: L07804. https://doi.org/10.1029/2010GL042675

[2] Bazelyan, E.M. and Raizer, Y.P. (1998) Spark Discharge. CRC Press, Boca Raton.

[3] Bazelyan, E.M. and Raizer, Y.P. (2000) Lightning Physics and Lightning Protection. CRC Press, Boca Raton. https://doi.org/10.1201/9780367801533

[4] Wescott, E.M., Sentman, D., Osborne, D., Hampton, D. and Heavner, M. (1995) Preliminary Results from the Sprites 94 Aircraft Campaign: 2. Blue Jets. Geophysical Research Letters, 22, 1209-1212. https://doi.org/10.1029/95GL00582

[5] Petrov, N.I. and Petrova, G.N. (1999) Physical Mechanisms for the Development of Lightning Discharges between a Thundercloud and the Ionosphere. Technical Physics, 44, 472-475. https://doi.org/10.1134/1.1259327

[6] Pasko, V.P. and George, J.J. (2002) Three-Dimensional Modeling of Blue Jets and Blue Starters. Journal of Geophysical Research: Space Physics, 107, SIA 12-1-SIA 12-16. https://doi.org/10.1029/2002JA009473

[7] Niemeyer, L., Ullrich, L. and Wiegart, N. (1989) The Mechanism of Leader Breakdown in Electronegative Gases. IEEE Transactions on Electrical Insulation, 24, 309-324. https://doi.org/10.1109/14.90289

[8] Raizer, Y.P., Milikh, G.M. and Shneider, M.N. (2006) On the Mechanism of Blue Jet Formation and Propagation. Geophysical Research Letters, 33, Article ID: L23801. https://doi.org/10.1029/2006GL027697

[9] Pancheshnyi, S.V., Starikovskaia, S.M. and Starikovskii, A.Y. (2001) Role of Photoionization Processes in Propagation of Cathode-Directed Streamer. Journal of Physics D: Applied Physics, 34, 105-115. https://doi.org/10.1088/0022-3727/34/1/317

[10] Babaeva, N.Y. and Naidis, G.V. (1996) Two-Dimensional Modelling of Positive Streamer Dynamics in Non-Uniform Electric Fields in Air. Journal of Physics $D$. Applied Physics, 29, 2423-2431. https://doi.org/10.1088/0022-3727/29/9/029

[11] Chen, S., Rong, Z. and Chijie, Z. (2013) The Diameters of Long Positive Streamers in Atmospheric Air under Lightning Impulse Voltage. Journal of Physics D: Applied 
Physics, 46, Article ID: 375203.

https://doi.org/10.1088/0022-3727/46/37/375203

[12] Pasko, V., Inan, U. and Bell, T. (1996a) Blue Jets Produced by Quasi-Electrostatic Pre-Discharge Thundercloud Fields. Geophysical Research Letters, 23, 301-304. https://doi.org/10.1029/96GL00149

[13] Sukhorukov, A.I., Mishin, E.V., Stubbe, P. and Rycroft, M.J. (1996) On Blue Jet Dynamics. Geophysical Research Letters, 23, 1625-1628. https://doi.org/10.1029/96GL01367

[14] Krehbiel, P.R., Riousset, J.A., Pasko, V.P., Thomas, R.J., Rison, W., Stanley, M.A. and Edens, H.E. (2008) Upward Electrical Discharges from Thunderstorms. Nature Geoscience, 1, 233-237. https://doi.org/10.1038/ngeo162

[15] Allen, N.L. and Ghaffar, A. (1995) The Conditions Required for Propagation of Cathode-Directed Positive Streamer in Air. Journal of Physics D: Applied Physics, 28, 331-337. https://doi.org/10.1088/0022-3727/28/2/016

[16] Kulikovsky, A.A. (1998) Positive Streamer in a Weak Field in Air: A Moving Avalanche-to-Streamer Transition. Physical Review E, 57, 7066-7074.

https://doi.org/10.1103/PhysRevE.57.7066

[17] Kulikovsky, A.A. (1997) Positive Streamer between Parallel Plate Electrodes in Atmospheric Pressure Air. Journal of Physics D: Applied Physics, 30, 441-450. https://doi.org/10.1088/0022-3727/30/3/017

[18] Nudnova, M.M. and Starikovskii, A.Y. (2008) Streamer Head Structure: Role of Ionization and Photoionization. Journal of Physics D: Applied Physics, 41, Article ID: 234003. https://doi.org/10.1088/0022-3727/41/23/234003

[19] Raizer, Y.P., Milikh, G.M. and Shneider, M.N. (2010) Streamerand Leader-Like Processes in the Upper Atmosphere: Models of Red Sprites and Blue Jets. Journal of Geophysical Research, 115, Article ID: A00E42.

https://doi.org/10.1029/2009JA014645 


\section{List of Symbols}

\begin{tabular}{cc}
\hline$K$ & Temperature in Kelvin unit \\
$E_{S}$ & Electric field inside the streamer channel \\
$N$ & Molecular number density at altitude $h$ \\
$h$ & Scaling factor of the order of $7 \mathrm{~km}$ \\
$R_{S}$ & Altitude above the round \\
$l_{\rho}$ & Radius of spherical streamer head \\
$E_{\max }$ & Thickness of space charge region \\
$n$ & Maximum electric field at the head of streamer \\
$L_{S}$ & A factor varying between 2 - 4 \\
$U_{C}$ & Length of streamer \\
$U_{L}$ & Cloud potential \\
$U_{O}$ & Peader tip potential \\
$R$ & Potential of leading front of streamer zone \\
$\varepsilon_{0}$ & Radius of spherical irregularity \\
\hline
\end{tabular}

